\title{
On Existence of Solutions of $q$-Perturbed Quadratic Integral Equations
}

\author{
Maryam Al-Yami \\ Department of Mathematics, Al Faisaliah Campus, Sciences Faculty, King Abdulaziz University, Jeddah, \\ Saudi Arabia \\ Email: malyami@kay.edu.sa
}

Received 18 April 2016; accepted 26 June 2016; published 29 June 2016

Copyright @ 2016 by authors and Scientific Research Publishing Inc.

This work is licensed under the Creative Commons Attribution International License (CC BY).

http://creativecommons.org/licenses/by/4.0/

(c) (i) Open Access

\section{Abstract}

We investigate a $q$-fractional integral equation with supremum and prove an existence theorem for it. We will prove that our $q$-integral equation has a solution in $C[0,1]$ which is monotonic on $[0,1]$. The monotonicity measures of noncompactness due to Banaś and Olszowy and Darbo's theorem are the main tools used in the proof of our main result.

\section{Keywords}

\author{
$q$-Fractional, Integral Equation, Monotonic Solutions, Darbo Theorem, Monotonicity Measure of \\ Noncompactness
}

\section{Introduction}

Jackson in [1] introduced the concept of quantum calculus ( $q$-calculus). This area of research has rich history and several applications, see [2]-[4] and references therein. There are several developments and applications of the $q$-calculus in mathematical physics, especially concerning quantum mechanics, the theory of relativity and special functions [5] [4]. Recently, several researchers attracted their attention by the concept of $q$-calculus, and we could find several new results in [6] [7] and the references therein.

In several papers among them [8]-[11], integral equations with nonsigular kernels have been studied. In [12][14] Darwish et al. introduced and studied the quadratic Volterra equations with supremum. Also, Banaś et al. and Darwish [13] [15]-[17] studied quadratic integral equations of arbitrary orders with singular kernels. In [18], Darwish generalized and extended Banaś et al. [15] results to the perturbed quadratic integral equations of arbitrary orders with singular kernels.

In this paper, we will study the $q$-perturbed quadratic integral equation with supremum 


$$
y(t)=f(t, y(t))+\frac{(\mathcal{A} y)(t)}{\Gamma_{q}(\beta)} \int_{0}^{t} k(t, s)(t-q s)^{(\beta-1)}(\mathcal{B} y)(s) \mathrm{d}_{q} s, t \in I=[0,1],
$$

where $0<\beta, q \in(0,1), f: I \times \mathbb{R} \rightarrow \mathbb{R}, \mathcal{A}, \mathcal{B}: C(I) \rightarrow C(I)$, and $k: I \times I \rightarrow \mathbb{R}$.

By using Darbo fixed point theorem and the monotonicity measure of noncompactness due to Banaś and Olszowy [19], we prove the existence of monotonic solution to Equation (1) in $C[0,1]$.

\section{2. $q$-Calculus and Measure of Noncompactness}

First, we collect basic definitions and results of the $q$-fractional integrals and $q$-derivatives, for more details, see [5] [6] [20] [21] and references therein.

First, for a real parameter $q \in(0,1)$, we define a $q$-real number $[a]_{q}$ by

$$
[a]_{q}=\frac{1-q^{a}}{1-q}, a \in \mathbb{R},
$$

and a $q$-analog of the Pochhammer symbol ( $q$-shifted factorial) is defined by

$$
(a ; q)_{n}= \begin{cases}1, & n=0, \\ \prod_{k=1}^{n-1}\left(1-a q^{k}\right), & n \in \mathbb{N} .\end{cases}
$$

Also, the $q$-analog of the power $(a-b)^{n}$ is given by

$$
(a-b)^{n}= \begin{cases}1, & n=0, \\ \prod_{k=1}^{n-1}\left(a-b q^{k}\right), & n \in \mathbb{N} ; a, b \in \mathbb{R} .\end{cases}
$$

Moreover,

$$
(a-b)^{(n)}=a^{n}(b / a ; q)_{n}, a \neq 0 .
$$

Notice that, $\lim _{n \rightarrow \infty}(a ; q)_{n}$ exists and we will denote it by $(a ; q)_{\infty}$.

More generally, for $\beta \in \mathbb{R}, a q^{\beta} \neq q^{-n}(n \in \mathbb{N})$, we define

$$
(a ; q)_{\beta}=\frac{(a ; q)_{\infty}}{\left(a q^{\beta} ; q\right)_{\infty}}
$$

and

$$
(a-b)^{(\beta)}=a^{\beta} \frac{(b / a ; q)_{\infty}}{\left(q^{\beta} b / a ; q\right)_{\infty}}
$$

Notice that $(a-b)^{(\beta)}=a^{\beta}(b / a ; q)_{\beta}$. Therefore, if $b=0$, then $a^{(\beta)}=a^{\beta}$.

The $q$-gamma function is defined by

$$
\Gamma_{q}(x)=\frac{G\left(q^{x}\right)}{(1-q)^{x-1} G(q)}, x \in \mathbb{R} \backslash\{0,-1,-2, \cdots\},
$$

where $G\left(q^{x}\right)=\frac{1}{\left(q^{x} ; q\right)_{\infty}}$. Or, equivalently, $\Gamma_{q}(x)=\frac{(1-q)^{(x-1)}}{(1-q)^{x-1}}$ and satisfies $\Gamma_{q}(x+1)=[x]_{q} \Gamma_{q}(x)$.

Next, the $q$-derivative of a function $f$ is given by

$$
\left(D_{q} f\right)(t)=\frac{f(t)-f(q t)}{t-q t},\left(D_{q} f\right)(0)=\lim _{t \rightarrow 0}\left(D_{q} f\right)(t),
$$

and the $q$-derivative of higher order of a function $f$ is defined by 


$$
\left(D_{q} f\right)(t)= \begin{cases}f(t), & n=0, \\ D_{q}\left(D_{q}^{n-1} f\right)(t), & n \in \mathbb{N} .\end{cases}
$$

The $q$-integral of a function $f$ defined on the interval $[0, b]$ is defined by

$$
\left(I_{q} f\right)(t)=\int_{a}^{t} f(s) \mathrm{d}_{q} s=t(1-q) \sum_{n=0}^{\infty} q^{n} f\left(t q^{n}\right), t \in[0, b] .
$$

If $f$ is given on the interval $[0, b]$ and $a \in[0, b]$ then

$$
\int_{a}^{b} f(s) \mathrm{d}_{q} s=\int_{0}^{b} f(s) \mathrm{d}_{q} s-\int_{0}^{a} f(s) \mathrm{d}_{q} s .
$$

The operator $I_{q}^{n}$ is defined by

$$
\left(I_{q}^{n} f\right)(t)= \begin{cases}f(t), & n=0 . \\ I_{q}\left(I_{q}^{n-1} f\right)(t), & n \in \mathbb{N},\end{cases}
$$

The fundamental theorem of calculus satisfies for $D_{q}$ and $I_{q}$, i.e., $\left(D_{q} I_{q} f\right)(t)=f(t)$, and if $f$ is continuous at $t=0$, then $\left(I_{q} D_{q} f\right)(t)=f(t)-f(0)$.

The following four formulas will be used later in this paper

$$
\begin{aligned}
& {[a(t-s)]^{(\beta)}=a^{\beta}(t-s)^{(\beta)}} \\
& { }_{t} D_{q}(t-s)^{(\beta)}=[\beta]_{q}(t-s)^{(\beta-1)} \\
& { }_{s} D_{q}(t-s)^{(\beta)}=-[\beta]_{q}(t-q s)^{(\beta-1)}
\end{aligned}
$$

and

$$
{ }_{t} D_{q} \int_{0}^{t} f(t, s) \mathrm{d}_{q} s=\int_{0}^{t} D_{q} f(t, s) \mathrm{d}_{q} s+f(q t, t),
$$

where ${ }_{t} D_{q}$ denotes the $q$-derivative with respect to variable $t$.

Notice that, if $\beta>0$ and $a \leq b \leq t$, then $(t-b)^{(\beta)} \leq(t-a)^{(\beta)}$.

Definition 1. [2] Let $f$ be a function defined on $[0,1]$. The fractional $q$-integral of the Riemann-Liouville type of order $\beta \geq 0$ is given by

$$
\left(I_{q}^{\beta} f\right)(t)= \begin{cases}f(t), & \beta=0, \\ \frac{1}{\Gamma_{q}(\beta)} \int_{0}^{t}(t-q s)^{(\beta-1)} f(s) \mathrm{d}_{q} s=t^{\beta}(1-q)^{\beta} \sum_{n=0}^{\infty} q^{n} \frac{\left(q^{\beta} ; q\right)_{n}}{(q ; q)_{n}} f\left(t q^{n}\right), & \beta>0, t \in[0,1] .\end{cases}
$$

Notice that, for $\beta=1$, the above $q$-integral reduces to (11).

Definition 2. [2] The fractional $q$-derivative of the Riemann-Liouville type of order $\beta \geq 0$ is given by

$$
\left(D_{q}^{\beta} f\right)(t)= \begin{cases}f(t), & \beta=0, \\ \left(D_{q}^{[\beta]} I_{q}^{[\beta]-\beta} f\right)(t), & \beta>0,\end{cases}
$$

where $[\beta]$ denotes the smallest integer greater than or equal to $\beta$.

In $q$-calculus, the derivative rule for the product of two functions and integration by parts formulas are

$$
\begin{aligned}
& \left(D_{q} f g\right)(t)=\left(D_{q} f\right)(t) g(t)+f(q t)\left(D_{q} g\right)(t), \\
& \int_{0}^{t} f(s)\left(D_{q} g\right)(s) \mathrm{d}_{q} s=[f(s) g(s)]_{0}^{t}-\int_{0}^{t}\left(D_{q} f\right)(s) g(q s) \mathrm{d}_{q} s .
\end{aligned}
$$

Lemma 1. Let $\gamma, \beta \geq 0$ and $f$ be a function defined on $[0,1]$. Then the following formulas are verified:

1) $\left(I_{q}^{\gamma} I_{q}^{\beta} f\right)(t)=\left(I_{q}^{\gamma+\beta} f\right)(t)$,

2) $\left(D_{q}^{\beta} I_{q}^{\beta} f\right)(t)=f(t)$. 
Lemma 2. [21] For $\beta>0$, using $q$-integration by parts, we have

$$
\left(I_{q}^{\beta} 1\right)(t)=\frac{t^{(\beta)}}{\Gamma_{q}(\beta+1)}
$$

or

$$
\int_{0}^{t}(t-q s)^{(\beta-1)} \mathrm{d}_{q} s=\frac{t^{(\beta)}}{[\beta]_{q}} .
$$

Second, we recall the basic concepts which we need throughout the paper about measure of noncompactness.

We assume that $(E,\|\cdot\|)$ is a real Banach space with zero element $\theta$ and we denote by $B(x, r)$ the closed ball with radius $r$ and centered $x$, where $B_{r} \equiv B(\theta, r)$.

Now, let $X \subset E$ and denote by $\bar{X}$ and Conv $X$ the closure and convex closure of $X$, respectively. Also, the symbols $X+Y$ and $\lambda X$ stands for the usual algebraic operators on sets.

Moreover, the families $\mathfrak{M}_{E}$ and $\mathfrak{N}_{E}$ are defined by $\mathfrak{M}_{E}=\{A \subset E: A \neq \varnothing, A$ is bounded $\}$ and $\mathfrak{N}_{E}=\left\{B \subset \mathfrak{M}_{E}: B\right.$ is relatively compact $\}$, respectively.

Definition 3. [22] Let $\mu: \mathfrak{M}_{E} \rightarrow \mathbb{R}_{+}$. If the following conditions

1) $\varnothing \neq\left\{X \in \mathfrak{M}_{E}: \mu(X)=0\right\}=\operatorname{ker} \mu \subset \mathfrak{N}_{E}$.

2) $X \subset Y$, then $\mu(X) \leq \mu(Y)$.

3) $\mu(X)=\mu(\bar{X})=\mu(\operatorname{Conv} X)$.

4) $\mu(\lambda X+(1-\lambda) Y) \leq \lambda \mu(X)+(1-\lambda) \mu(Y), 0 \leq \lambda \leq 1$ and

5) if $\left(X_{n}\right)$ is a sequence of closed subsets of $\mathfrak{M}_{E}$ with $X_{n+1} \subset X_{n}, n=1,2,3, \cdots$, and $\lim _{n \rightarrow \infty}\left(X_{n}\right)=0$ then $X_{\infty}=\bigcap_{n=1}^{\infty} X_{n} \neq \varnothing$ hold. Then, the mapping $\mu$ is said to be a measure of noncompactness in $E$.

Here, $\operatorname{ker} \mu$ is the kernel of the measure of noncompactness $\mu$.

Our result will establish in $C(I)$ the Banach space of all defined, continuous and real functions on $I \equiv[0,1]$ with $\|y\|=\max _{t \in I}|y(t)|$.

Next, we defined the measure of noncompactness related to monotonicity in $C(I)$, see [19] [22].

We fix a bounded subset $Y \neq \varnothing$ of $C(I)$. For $\varepsilon \geq 0$ and $y \in Y, \omega(y, \varepsilon)$ denotes the modulus of continuity of the function $y$ given by

$$
\omega(y, \varepsilon)=\sup \{|y(t)-y(s)|: t, s \in I,|t-s| \leq \varepsilon\}
$$

Moreover, we let

$$
\omega(Y, \varepsilon)=\sup \{\omega(y, \varepsilon): y \in Y\}
$$

and

$$
\omega_{0}(Y)=\lim _{\varepsilon \rightarrow 0} \omega(Y, \varepsilon) .
$$

Define

$$
d(y)=\sup _{s, t \in I, s \leq t}(|y(t)-y(s)|-[y(t)-y(s)])
$$

and

$$
d(Y)=\sup _{y \in Y} d(y) .
$$

Notice that, all functions in $Y$ are nondecreasing on $I$ if and only if $d(Y)=0$.

Now, we define the map $\mu$ on $\mathfrak{M}_{C(I)}$ as

$$
\mu(Y)=d(Y)+\omega_{0}(Y) .
$$


Clearly, $\mu$ verifies all conditions in Definition 3 and, therefore it is a measure of noncompactness in $C(I)$ [19].

Definition 4.Let $\varnothing \neq \Omega \subset E$. Let $\mathcal{P}: \Omega \rightarrow E$ be a continuous operator. Suppose that $\mathcal{P}$ maps bounded sets onto bounded ones. If there exists a bounded $Y \subset \Omega$ with $\mu(\mathcal{P} Y) \leq \gamma \mu(Y), \gamma \geq 0$, then $\mathcal{P}$ is said to be satisfies the Darbo condition with respect to a measure of noncompactness $\mu$.

If $\gamma<1$, then $\mathcal{P}$ is called a contraction operator with respect to $\mu$.

Theorem 1. [23] Let $Q \neq \varnothing$ be a bounded, convex and closed subset of $E$. If $\mathcal{P}: Q \rightarrow Q$ is a Contraction operator with respect to $\mu$. Then $\mathcal{P}$ has at least one fixed point belongs to $Q$.

\section{Existence Theorem}

Let us consider the following suggestions:

$\left.a_{1}\right) f: I \times \mathbb{R} \rightarrow \mathbb{R}$ is continuous and

$$
\exists 0 \leq c<1 \text { s.t. }|f(t, y)-f(t, x)| \leq c|y-x| \forall t \in I \text { and } x, y \in \mathbb{R}
$$

Moreover, $f: I \times \mathbb{R}_{+} \rightarrow \mathbb{R}$ and $f^{*}=\max _{t \in I} f(t, 0)$.

$a_{2}$ ) The superposition operator $F$ generated by the function $f$ satisfies for any nonnegative function $y$ the condition $d(F y) \leq c d(y)$, where $\mathrm{c}$ is the same constant as in $\left.a_{1}\right)$.

$\left.a_{3}\right) \quad \mathcal{A}: C(I) \rightarrow C(I)$ is a continuous operator which satisfies the Darbo condition for the measure of noncompactness $\mu$ with a constant $\eta$. Also, $\mathcal{A} y \geq 0$ if $y \geq 0$.

$\left.a_{4}\right) \exists a, b \geq 0$, s.t. $|(\mathcal{A} y)(t)| \leq a+\|y\| \forall y \in C(I), t \in I$.

$a_{5}$ ) The function $k: I \times I \rightarrow \mathbb{R}_{+}$is continuous on $I \times I$ and nondecreasing $\forall t$ and $s$ separately. Moreover, $k^{*}=\sup _{(t, s) \in I \times I} k(t, s)$.

$\left.a_{6}\right) \mathcal{B}: C(I) \rightarrow C(I)$ is a continuous operator and there is a nondecreasing function $\phi: \mathbb{R}_{+} \rightarrow \mathbb{R}_{+}$such that $\|\mathcal{B} y\| \leq \phi(\|y\|)$ for any $y \in C(I)$. Moreover, for every function $y \in C(I)$ which is nonnegative on $I$, the function $\mathcal{B} y$ is nonnegative and nondecreasing on $I$.

$\left.a_{7}\right) \exists r_{0}>0$ such that

$$
f^{*}+c r+\frac{(a+b r) k^{*} \phi(r)}{\Gamma_{q}(\beta+1)} \leq r
$$

and $c+\frac{\eta k^{*} \phi\left(r_{0}\right)}{\Gamma_{q}(\beta+1)}<1$ lows

Before, we state and prove our main theorem, we define the two operators $\mathcal{K}$ and $\mathcal{T}$ on $C(I)$ as fol-

$$
(\mathcal{K} y)(t)=\frac{1}{\Gamma_{q}(\beta)} \int_{0}^{t} k(t, s)(t-q s)^{(\beta-1)}(\mathcal{B} y)(s) \mathrm{d} s
$$

and

$$
(\mathcal{T} y)(t)=f(t, y(t))+(\mathcal{A} y)(t)(\mathcal{K} y)(t)
$$

respectively. Finding a fixed point of the operator $\mathcal{T}$ defined on the space $C(I)$ is equivalent to solving Equation (1).

Theorem 2. Assume the suggestions $\left(a_{1}\right)-\left(a_{7}\right)$ be verified, then Equation (1) has at least one solution $y \in C(I)$ which is nondecreasing on $I$.

Proof. We divide the proof into seven steps for better readability.

Step 1: We will show that the operator $\mathcal{T}$ maps $C(I)$ into itself.

For this, it is sufficient to show that $\mathcal{K} y \in C(I)$ if $y \in C(I)$. Fix $\varepsilon>0$ and let $y \in C(I)$ and $t_{1}, t_{2} \in I\left(t_{1} \leq t_{2}\right)$ with $\left|t_{2}-t_{1}\right| \leq \varepsilon$. We have 


$$
\begin{aligned}
& \left|(\mathcal{K} y)\left(t_{2}\right)-(\mathcal{K} y)\left(t_{1}\right)\right| \\
& =\frac{1}{\Gamma_{q}(\beta)}\left|\int_{0}^{t_{2}} k\left(t_{2}, s\right)\left(t_{2}-q s\right)^{(\beta-1)}(\mathcal{B} y)(s) \mathrm{d}_{q} s-\int_{0}^{t_{1}} k\left(t_{1}, s\right)\left(t_{1}-q s\right)^{(\beta-1)}(\mathcal{B} y)(s) \mathrm{d}_{q} s\right| \\
& \leq \frac{1}{\Gamma_{q}(\beta)}\left|\int_{0}^{t_{2}} k\left(t_{2}, s\right)\left(t_{2}-q s\right)^{(\beta-1)}(\mathcal{B} y)(s) \mathrm{d}_{q} s-\int_{0}^{t_{2}} k\left(t_{1}, s\right)\left(t_{2}-q s\right)^{(\beta-1)}(\mathcal{B} y)(s) \mathrm{d}_{q} s\right| \\
& +\frac{1}{\Gamma_{q}(\beta)}\left|\int_{0}^{t_{2}} k\left(t_{1}, s\right)\left(t_{2}-q s\right)^{(\beta-1)}(\mathcal{B} y)(s) \mathrm{d}_{q} s-\int_{0}^{t_{1}} k\left(t_{1}, s\right)\left(t_{2}-q s\right)^{(\beta-1)}(\mathcal{B} y)(s) \mathrm{d}_{q} s\right| \\
& +\frac{1}{\Gamma_{q}(\beta)}\left|\int_{0}^{t_{1}} k\left(t_{1}, s\right)\left(t_{2}-q s\right)^{(\beta-1)}(\mathcal{B} y)(s) \mathrm{d}_{q} s-\int_{0}^{t_{1}} k\left(t_{1}, s\right)\left(t_{1}-q s\right)^{(\beta-1)}(\mathcal{B} y)(s) \mathrm{d}_{q} s\right| \\
& \leq \frac{1}{\Gamma_{q}(\beta)} \int_{0}^{t_{2}}\left|k\left(t_{2}, s\right)-k\left(t_{1}, s\right)\right|\left(t_{2}-q s\right)^{(\beta-1)}|(\mathcal{B y})(s)| \mathrm{d}_{q} s \\
& +\frac{1}{\Gamma_{q}(\beta)} \int_{t_{1}}^{t_{2}}\left|k\left(t_{1}, s\right)\right|\left(t_{2}-q s\right)^{(\beta-1)}|(\mathcal{B} y)(s)| \mathrm{d}_{q} s \\
& +\frac{1}{\Gamma_{q}(\beta)} \int_{0}^{t_{1}}\left|k\left(t_{1}, s\right)\right|\left[\left(t_{1}-q s\right)^{(\beta-1)}-\left(t_{2}-q s\right)^{(\beta-1)}\right]|(\mathcal{B} y)(s)| \mathrm{d}_{q} s \\
& \leq \frac{\phi(\|y\|) \omega_{k}(\varepsilon, .)}{\Gamma_{q}(\beta)} \int_{0}^{t_{2}}\left(t_{2}-q s\right)^{(\beta-1)} \mathrm{d}_{q} s \\
& +\frac{k^{*} \phi(\|y\|)}{\Gamma_{q}(\beta)}\left\{\int_{0}^{t_{1}}\left[\left(t_{1}-q s\right)^{(\beta-1)}-\left(t_{2}-q s\right)^{(\beta-1)}\right] \mathrm{d}_{q} s+\int_{t_{1}}^{t_{2}}\left(t_{2}-q s\right)^{(\beta-1)} \mathrm{d}_{q} s\right\} \\
& =\frac{\phi(\|y\|) \omega_{k}(\varepsilon, .)}{\Gamma_{q}(\beta+1)} t_{2}^{(\beta)}+\frac{k^{*} \phi(\|y\|)}{\Gamma_{q}(\beta+1)}\left[t_{1}^{(\beta)}-t_{2}^{(\beta)}+2\left(t_{2}-t_{1}\right)^{(\beta)}\right] \\
& \leq \frac{\phi(\|y\|) \omega_{k}(\varepsilon, .)}{\Gamma_{q}(\beta+1)} t_{2}^{\beta}+\frac{2 k^{*} \phi(\|y\|)}{\Gamma_{q}(\beta+1)}\left(t_{2}-t_{1}\right)^{(\beta)} \\
& \leq \frac{\phi(\|y\|) \omega_{k}(\varepsilon, .)}{\Gamma_{q}(\beta+1)}+\frac{2 k^{*} \phi(\|y\|)}{\Gamma_{q}(\beta+1)} \varepsilon^{\beta} .
\end{aligned}
$$

Notice that, we have used

$$
\omega_{k}(\varepsilon, .)=\sup \{|k(t, s)-k(\tau, s)|: t, s, \tau \in I \text { and }|t-\tau| \leq \varepsilon\} .
$$

Notice that, since the function $k$ is uniformly continuous on $I \times I$, then when $\varepsilon \rightarrow 0$ we have that $\omega_{k}(\varepsilon,.) \rightarrow 0$.

Thus $\mathcal{K} y \in C(I)$, and therefore, $\mathcal{T} y \in C(I)$.

Step 2: $\mathcal{T}$ applies $B_{r_{0}}$ into itself.

Now, $\forall t \in I$, we have

$$
\begin{aligned}
|(\mathcal{T} y)(t)| & \leq\left|f(t, y(t))+\frac{(\mathcal{A} y)(t)}{\Gamma_{q}(\beta)} \int_{0}^{t} k(t, s)(t-q s)^{(\beta-1)}(\mathcal{B} y)(s) \mathrm{d}_{q} s\right| \\
& \leq|f(t, y(t))-f(t, 0)|+|f(t, 0)|+\frac{|(\mathcal{A} y)(t)|}{\Gamma_{q}(\beta)} \int_{0}^{t}|k(t, s)|(t-q s)^{(\beta-1)}|(\mathcal{B} y)(s)| \mathrm{d}_{q} s \\
& \leq c\|y\|+f^{*}+\frac{(a+b\|y\|) k^{*} \phi(\|y\|)}{\Gamma_{q}(\beta)} \int_{0}^{t}(t-q s)^{(\beta-1)} \mathrm{d}_{q} s \\
& =c\|y\|+f^{*}+\frac{(a+b\|y\|) k^{*} \phi(\|y\|)}{\Gamma_{q}(\beta+1)}
\end{aligned}
$$


Hence

$$
\|\mathcal{T} y\| \leq c\|y\|+f^{*}+\frac{(a+b\|y\|) k^{*} \phi(\|y\|)}{\Gamma_{q}(\beta+1)} .
$$

Therefore, if $\|y\| \leq r_{0}$ we get from assumption $a_{7}$ ) the following

$$
\|\mathcal{T} y\| \leq c r_{0}+f^{*}+\frac{\left(a+b r_{0}\right) k^{*} \phi\left(r_{0}\right)}{\Gamma_{q}(\beta+1)} \leq r_{0} .
$$

Therefore, $\mathcal{T}$ maps $B_{r_{0}}$ into itself.

We define the subset $B_{r_{0}}^{+}$of $B_{r_{0}}$ by

$$
B_{r_{0}}^{+}=\left\{y \in B_{r_{0}}: y(t) \geq 0, \text { for } t \in I\right\}
$$

It is clear that $B_{r_{0}}^{+} \neq \varnothing$ is closed, convex and bounded.

Step 3: $\mathcal{T}$ applies the set $B_{r_{0}}^{+}$into itself.

By this facts and suggestions $a_{1}$ ), $a_{4}$ ) and $a_{6}$ ), we obtain $\mathcal{T}$ transforms $B_{r_{0}}^{+}$into itself.

Step 4: The operator $\mathcal{T}$ is continuous on $B_{r_{0}}^{+}$.

To prove this, we fix $\left(y_{n}\right)$ to be a sequence in $B_{r_{0}}^{+}$with $y_{n} \rightarrow y$. We will show that $\mathcal{T}_{n} \rightarrow \mathcal{T} y$.

Thus, we have $\forall t \in I$,

$$
\begin{aligned}
& \left|\left(\mathcal{T}_{n}\right)(t)-(\mathcal{T} y)(t)\right| \leq\left|f\left(t, y_{n}(t)\right)-f(t, y(t))\right| \\
& \quad+\left|\frac{\left(\mathcal{A} y_{n}\right)(t)}{\Gamma_{q}(\beta)} \int_{0}^{t} k(t, s)(t-q s)^{(\beta-1)}\left(\mathcal{B} y_{n}\right)(s) \mathrm{d}_{q} s-\frac{(\mathcal{A} y)(t)}{\Gamma_{q}(\beta)} \int_{0}^{t} k(t, s)(t-q s)^{(\beta-1)}(\mathcal{B} y)(s) \mathrm{d}_{q} s\right| \\
& \leq c\left|y_{n}(t)-y(t)\right|+\left|\frac{\left(\mathcal{A} y_{n}\right)(t)}{\Gamma_{q}(\beta)} \int_{0}^{t} k(t, s)(t-q s)^{(\beta-1)}\left(\mathcal{B} y_{n}\right)(s) \mathrm{d}_{q} s-\frac{(\mathcal{A} y)(t)}{\Gamma_{q}(\beta)} \int_{0}^{t} k(t, s)(t-q s)^{(\beta-1)}\left(\mathcal{B} y_{n}\right)(s) \mathrm{d}_{q} s\right| \\
& \quad+\left|\frac{(\mathcal{A} y)(t)}{\Gamma_{q}(\beta)} \int_{0}^{t} k(t, s)(t-q s)^{(\beta-1)}\left(\mathcal{B} y_{n}\right)(s) \mathrm{d}_{q} s-\frac{(\mathcal{A} y)(t)}{\Gamma_{q}(\beta)} \int_{0}^{t} k(t, s)(t-q s)^{(\beta-1)}(\mathcal{B} y)(s) \mathrm{d}_{q} s\right| \\
& \leq c\left|y_{n}(t)-y(t)\right|+\frac{\left|\left(\mathcal{A} y_{n}\right)(t)-(\mathcal{A} y)(t)\right|}{\Gamma_{q}(\beta)} \int_{0}^{t}|k(t, s)|(t-q s)^{(\beta-1)}\left|\left(\mathcal{B} y_{n}\right)(s)\right| \mathrm{d}_{q} s \\
& \quad+\frac{|(\mathcal{A} y)(t)|}{\Gamma_{q}(\beta)} \int_{0}^{t}|k(t, s)|(t-q s)^{(\beta-1)}\left|\left(\mathcal{B} y_{n}\right)(s)-(\mathcal{B} y)(s)\right| \mathrm{d}_{q} s .
\end{aligned}
$$

Consequently,

$$
\left\|\mathcal{T}_{n}-\mathcal{T} y\right\| \leq c\left\|y_{n}-y\right\|+\frac{k^{*} \phi\left(r_{0}\right)\left\|\mathcal{A} y_{n}-\mathcal{A} y\right\|}{\Gamma_{q}(\beta+1)}+\frac{\left(a+b r_{0}\right) k^{*}\left\|\mathcal{B} y_{n}-\mathcal{B} y\right\|}{\Gamma_{q}(\beta+1)} .
$$

As $\mathcal{A}$ and $\mathcal{B}$ are continuous operators, $\exists n_{1} \in \mathbb{N}$ such that

$$
\left\|\mathcal{A} y_{n}-\mathcal{A} y\right\| \leq \frac{\varepsilon \Gamma_{q}(\beta+1)}{3 k^{*} \phi\left(r_{0}\right)}, \forall n \geq n_{1} .
$$

Also, $\exists n_{2} \in \mathbb{N}$ such that

$$
\left\|\mathcal{B} y_{n}-\mathcal{B} y\right\| \leq \frac{\varepsilon \Gamma_{q}(\beta+1)}{3 k^{*}\left(a+b r_{0}\right)}, \forall n \geq n_{2} .
$$

Furthermore, $\exists n_{3} \in \mathbb{N}$ such that

$$
\left\|y_{n}-y\right\| \leq \frac{\varepsilon}{3 c}, \quad \forall n \geq n_{3} .
$$


Now, take $\max \left\{n_{1}, n_{2}, n_{3}\right\} \leq n$, then (38) gives us that

$$
\left\|\mathcal{T}_{n}-\mathcal{T} y\right\| \leq \varepsilon .
$$

This shows that $\mathcal{T}$ is continuous in $B_{r_{0}}^{+}$.

Step 5: In recognition of $\mathcal{T}$ with respect to the quantity $\omega_{0}$.

Now, we take $\varnothing \neq Y \subset B_{r_{0}}^{+}$. Let us fix an arbitrarily number $\varepsilon>0$ and choose $y \in Y$ and $t_{1}, t_{2} \in I$ with $\left|t_{2}-t_{1}\right| \leq \varepsilon$. We will be supposed that $t_{1} \leq t_{2}$ because no generality will be loss. Then, by using our suggestions and inequality (31), we get

$$
\begin{aligned}
\mid & (\mathcal{T} y)\left(t_{2}\right)-(\mathcal{T} y)\left(t_{1}\right) \mid \\
\leq & \left|f\left(t_{2}, y\left(t_{2}\right)\right)-f\left(t_{1}, y\left(t_{1}\right)\right)\right|+\left|(\mathcal{A} y)\left(t_{2}\right)(\mathcal{K} y)\left(t_{2}\right)-(\mathcal{A} y)\left(t_{2}\right)(\mathcal{K} y)\left(t_{1}\right)\right| \\
& +\left|(\mathcal{A} y)\left(t_{2}\right)(\mathcal{K} y)\left(t_{1}\right)-(\mathcal{A} y)\left(t_{1}\right)(\mathcal{K} y)\left(t_{1}\right)\right| \\
\leq & \left|f\left(t_{2}, y\left(t_{2}\right)\right)-f\left(t_{1}, y\left(t_{2}\right)\right)\right|+\left|f\left(t_{1}, y\left(t_{2}\right)\right)-f\left(t_{1}, y\left(t_{1}\right)\right)\right| \\
& +\left|(\mathcal{A} y)\left(t_{2}\right)\right|\left|(\mathcal{K} y)\left(t_{2}\right)-(\mathcal{K} y)\left(t_{1}\right)\right|+\left|(\mathcal{A} y)\left(t_{2}\right)-(\mathcal{A} y)\left(t_{1}\right)\right|\left|(\mathcal{K} y)\left(t_{1}\right)\right| \\
\leq & \gamma_{r_{0}}(f, \varepsilon)+c \omega(y, \varepsilon)+\left|(\mathcal{A} y)\left(t_{2}\right)\right|\left|(\mathcal{K} y)\left(t_{2}\right)-(\mathcal{K} y)\left(t_{1}\right)\right| \\
& +\left|(\mathcal{A} y)\left(t_{2}\right)-(\mathcal{A} y)\left(t_{1}\right)\right|\left|(\mathcal{K} y)\left(t_{1}\right)\right| \\
\leq & \gamma_{r_{0}}(f, \varepsilon)+c \omega(y, \varepsilon)+\frac{(a+b\|y\|) \phi(\|y\|)}{\Gamma_{q}(\beta+1)}\left[\omega_{k}(\varepsilon, .)+2 k^{*} \varepsilon^{\beta}\right] \\
& +\frac{\omega(\mathcal{A} y, \varepsilon)}{\Gamma_{q}(\beta+1)} k^{*} \phi(\|y\|) \\
\leq & \gamma_{r_{0}}(f, \varepsilon)+c \omega(y, \varepsilon)+\frac{\left(a+b r_{0}\right) \phi\left(r_{0}\right)}{\Gamma_{q}(\beta+1)}\left[\omega_{k}(\varepsilon, .)+2 k^{*} \varepsilon^{\beta}\right] \\
& +\frac{\omega(\mathcal{A} y, \varepsilon)}{\Gamma_{q}(\beta+1)} k^{*} \phi\left(r_{0}\right) .
\end{aligned}
$$

The last estimate implies

$$
\begin{aligned}
\omega(\mathcal{T} y, \varepsilon) & \leq \gamma_{r_{0}}(f, \varepsilon)+c \omega(y, \varepsilon) \\
& +\frac{\left(a+b r_{0}\right) \phi\left(r_{0}\right)}{\Gamma_{q}(\beta+1)}\left[\omega_{k}(\varepsilon, .)+2 k^{*} \varepsilon^{\beta}\right]+\frac{\omega(\mathcal{A y}, \varepsilon)}{\Gamma_{q}(\beta+1)} k^{*} \phi\left(r_{0}\right)
\end{aligned}
$$

and, consequently,

$$
\begin{aligned}
\omega(\mathcal{T Y}, \varepsilon) & \leq \gamma_{r_{0}}(f, \varepsilon)+c \omega(Y, \varepsilon) \\
& +\frac{\left(a+b r_{0}\right) \phi\left(r_{0}\right)}{\Gamma_{q}(\beta+1)}\left[\omega_{k}(\varepsilon, .)+2 k^{*} \varepsilon^{\beta}\right]+\frac{\omega(\mathcal{A} Y, \varepsilon)}{\Gamma_{q}(\beta+1)} k^{*} \phi\left(r_{0}\right) .
\end{aligned}
$$

Since the function $k$ is uniformly continuous on $I \times I$ and the function $f$ is continuous on $I \times\left[0, r_{0}\right]$, then the last inequality gives us that

$$
\omega_{0}(\mathcal{T} Y) \leq c \omega_{0}(Y)+\frac{k^{*} \phi\left(r_{0}\right)}{\Gamma_{q}(\beta+1)} \omega_{0}(\mathcal{A} Y) .
$$

Step 6: In recognition of $\mathcal{T}$ with respect to the quantity $d$.

Here, we fix an arbitrary $y \in Y$ and $t_{1}, t_{2} \in I$ with $t_{2}>t_{1}$. Then, by our assumption, we obtain our suggestions, we have 


$$
\begin{aligned}
& \left|(\mathcal{T} y)\left(t_{2}\right)-(\mathcal{T} y)\left(t_{1}\right)\right|-\left[(\mathcal{T} y)\left(t_{2}\right)-(\mathcal{T} y)\left(t_{1}\right)\right] \\
& =\mid f\left(t_{2}, y\left(t_{2}\right)\right)+\frac{(\mathcal{A} y)\left(t_{2}\right)}{\Gamma_{q}(\beta)} \int_{0}^{t_{2}} k\left(t_{2}, s\right)\left(t_{2}-q s\right)^{(\beta-1)}(\mathcal{B} y)(s) \mathrm{d}_{q} s \\
& -f\left(t_{1}, y\left(t_{1}\right)\right)-\frac{(\mathcal{A} y)\left(t_{1}\right)}{\Gamma_{q}(\beta)} \int_{0}^{t_{1}} k\left(t_{1}, s\right)\left(t_{1}-q s\right)^{(\beta-1)}(\mathcal{B} y)(s) \mathrm{d}_{q} s \\
& -\left[f\left(t_{2}, y\left(t_{2}\right)\right)+\frac{(\mathcal{A} y)\left(t_{2}\right)}{\Gamma_{q}(\beta)} \int_{0}^{t_{2}} k\left(t_{2}, s\right)\left(t_{2}-q s\right)^{(\beta-1)}(\mathcal{B} y)(s) \mathrm{d}_{q} s\right. \\
& \left.-f\left(t_{1}, y\left(t_{1}\right)\right)-\frac{(\mathcal{A} y)\left(t_{1}\right)}{\Gamma_{q}(\beta)} \int_{0}^{t_{1}} k\left(t_{1}, s\right)\left(t_{1}-q s\right)^{(\beta-1)}(\mathcal{B y})(s) \mathrm{d}_{q} s\right] \\
& \leq\left\{\left|f\left(t_{2}, y\left(t_{2}\right)\right)-f\left(t_{1}, y\left(t_{1}\right)\right)\right|-\left[f\left(t_{2}, y\left(t_{2}\right)\right)-f\left(t_{1}, y\left(t_{1}\right)\right)\right]\right\} \\
& +\left|\frac{(\mathcal{A} y)\left(t_{2}\right)}{\Gamma_{q}(\beta)} \int_{0}^{t_{2}} k\left(t_{2}, s\right)\left(t_{2}-q s\right)^{(\beta-1)}(\mathcal{B} y)(s) \mathrm{d}_{q} s-\frac{(T x)\left(t_{1}\right)}{\Gamma_{q}(\beta)} \int_{0}^{t_{2}} k\left(t_{2}, s\right)\left(t_{2}-q s\right)^{(\beta-1)}(\mathcal{B} y)(s) \mathrm{d}_{q} s\right| \\
& +\left|\frac{(\mathcal{A} y)\left(t_{1}\right)}{\Gamma_{q}(\beta)} \int_{0}^{t_{2}} k\left(t_{2}, s\right)\left(t_{2}-q s\right)^{(\beta-1)}(\mathcal{B} y)(s) \mathrm{d}_{q} s-\frac{(T x)\left(t_{1}\right)}{\Gamma_{q}(\beta)} \int_{0}^{t_{1}} k\left(t_{1}, s\right)\left(t_{1}-q s\right)^{(\beta-1)}(\mathcal{B} y)(s) \mathrm{d}_{q} s\right| \\
& -\left\{\left[\frac{(\mathcal{A} y)\left(t_{2}\right)}{\Gamma_{q}(\beta)} \int_{0}^{t_{2}} k\left(t_{2}, s\right)\left(t_{2}-q s\right)^{(\beta-1)}(\mathcal{B y})(s) \mathrm{d}_{q} s-\frac{(T x)\left(t_{1}\right)}{\Gamma_{q}(\beta)} \int_{0}^{t_{2}} k\left(t_{2}, s\right)\left(t_{2}-q s\right)^{(\beta-1)}(\mathcal{B y})(s) \mathrm{d}_{q} s\right]\right. \\
& \left.+\left[\frac{(\mathcal{A} y)\left(t_{1}\right)}{\Gamma_{q}(\beta)} \int_{0}^{t_{2}} k\left(t_{2}, s\right)\left(t_{2}-q s\right)^{(\beta-1)}(\mathcal{B} y)(s) \mathrm{d}_{q} s-\frac{(T x)\left(t_{1}\right)}{\Gamma_{q}(\beta)} \int_{0}^{t_{1}} k\left(t_{1}, s\right)\left(t_{1}-q s\right)^{(\beta-1)}(\mathcal{B} y)(s) \mathrm{d}_{q} s\right]\right\} \\
& \leq\left\{\left|f\left(t_{2}, y\left(t_{2}\right)\right)-f\left(t_{1}, y\left(t_{1}\right)\right)\right|-\left[f\left(t_{2}, y\left(t_{2}\right)\right)-f\left(t_{1}, y\left(t_{1}\right)\right)\right]\right\} \\
& +\left\{\left|(\mathcal{A} y)\left(t_{2}\right)-(\mathcal{A} y)\left(t_{1}\right)\right|-\left[(\mathcal{A} y)\left(t_{2}\right)-(\mathcal{A} y)\left(t_{1}\right)\right]\right\} \frac{1}{\Gamma_{q}(\beta)} \int_{0}^{t_{2}} k\left(t_{2}, s\right)\left(t_{2}-q s\right)^{(\beta-1)}(\mathcal{B} y)(s) \mathrm{d}_{q} s \\
& +\frac{(\mathcal{A} y)\left(t_{1}\right)}{\Gamma_{q}(\beta)}\left\{\left|\int_{0}^{t_{2}} k\left(t_{2}, s\right)\left(t_{2}-q s\right)^{(\beta-1)}(\mathcal{B} y)(s) \mathrm{d}_{q} s-\int_{0}^{t_{1}} k\left(t_{1}, s\right)\left(t_{1}-q s\right)^{(\beta-1)}(\mathcal{B} y)(s) \mathrm{d}_{q} s\right|\right. \\
& \left.-\left[\int_{0}^{t_{2}} k\left(t_{2}, s\right)\left(t_{2}-q s\right)^{(\beta-1)}(\mathcal{B} y)(s) \mathrm{d}_{q} s-\int_{0}^{t_{1}} k\left(t_{1}, s\right)\left(t_{1}-q s\right)^{(\beta-1)}(\mathcal{B} y)(s) \mathrm{d}_{q} s\right]\right\} .
\end{aligned}
$$

Now, we will prove that

$$
\int_{0}^{t_{2}} k\left(t_{2}, s\right)\left(t_{2}-q s\right)^{(\beta-1)}(\mathcal{B} y)(s) \mathrm{d}_{q} s-\int_{0}^{t_{1}} k\left(t_{1}, s\right)\left(t_{1}-q s\right)^{(\beta-1)}(\mathcal{B} y)(s) \mathrm{d}_{q} s \geq 0 .
$$

We find that

$$
\begin{aligned}
& \int_{0}^{t_{2}} k\left(t_{2}, s\right)\left(t_{2}-q s\right)^{(\beta-1)}(\mathcal{B} y)(s) \mathrm{d}_{q} s-\int_{0}^{t_{1}} k\left(t_{1}, s\right)\left(t_{1}-q s\right)^{(\beta-1)}(\mathcal{B y})(s) \mathrm{d}_{q} s \\
& =\int_{0}^{t_{2}} k\left(t_{2}, s\right)\left(t_{2}-q s\right)^{(\beta-1)}(\mathcal{B} y)(s) \mathrm{d}_{q} s-\int_{0}^{t_{2}} k\left(t_{1}, s\right)\left(t_{2}-q s\right)^{(\beta-1)}(\mathcal{B} y)(s) \mathrm{d}_{q} s \\
& \quad+\int_{0}^{t_{2}} k\left(t_{1}, s\right)\left(t_{2}-q s\right)^{(\beta-1)}(\mathcal{B} y)(s) \mathrm{d}_{q} s-\int_{0}^{t_{1}} k\left(t_{1}, s\right)\left(t_{2}-q s\right)^{(\beta-1)}(\mathcal{B y})(s) \mathrm{d}_{q} s \\
& \quad+\int_{0}^{t_{1}} k\left(t_{1}, s\right)\left(t_{2}-q s\right)^{(\beta-1)}(\mathcal{B} y)(s) \mathrm{d}_{q} s-\int_{0}^{t_{1}} k\left(t_{1}, s\right)\left(t_{1}-q s\right)^{(\beta-1)}(\mathcal{B} y)(s) \mathrm{d}_{q} s \\
& =\int_{0}^{t_{2}}\left(k\left(t_{2}, s\right)-k\left(t_{1}, s\right)\right)\left(t_{2}-q s\right)^{(\beta-1)}(\mathcal{B} y)(s) \mathrm{d}_{q} s+\int_{t_{1}}^{t_{2}} k\left(t_{1}, s\right)\left(t_{2}-q s\right)^{(\beta-1)}(\mathcal{B y})(s) \mathrm{d}_{q} s \\
& \quad+\int_{0}^{t_{1}} k\left(t_{1}, s\right)\left[\left(t_{2}-q s\right)^{(\beta-1)}-\left(t_{1}-q s\right)^{(\beta-1)}\right](\mathcal{B} y)(s) \mathrm{d}_{q} s .
\end{aligned}
$$


But, $k\left(t_{1}, s\right) \leq k\left(t_{2}, s\right)$ because $k(t, s)$ is increasing with respect to $t$, then

$$
\int_{0}^{t_{2}}\left(k\left(t_{2}, s\right)-k\left(t_{1}, s\right)\right)\left(t_{2}-q s\right)^{(\beta-1)}(\mathcal{B} y)(s) \mathrm{d}_{q} s \geq 0,
$$

and, since $\left(t_{2}-q s\right)^{(\beta-1)}-\left(t_{1}-q s\right)^{(\beta-1)}$ is negative for $s \in\left[0, t_{1}\right)$ then

$$
\begin{aligned}
& \int_{0}^{t_{1}} k\left(t_{1}, s\right)\left[\left(t_{2}-q s\right)^{(\beta-1)}-\left(t_{1}-q s\right)^{(\beta-1)}\right](\mathcal{B} y)(s) \mathrm{d}_{q} s+\int_{t_{1}}^{t_{2}} k\left(t_{1}, s\right)\left(t_{2}-q s\right)^{(\beta-1)}(\mathcal{B} y)(s) \mathrm{d}_{q} s \\
& \geq \int_{0}^{t_{1}} k\left(t_{1}, t_{1}\right)\left[\left(t_{2}-q s\right)^{(\beta-1)}-\left(t_{1}-q s\right)^{(\beta-1)}\right](\mathcal{B} y)\left(t_{1}\right) \mathrm{d}_{q} s+\int_{t_{1}}^{t_{2}} k\left(t_{1}, t_{1}\right)\left(t_{2}-q s\right)^{(\beta-1)}(\mathcal{B} y)\left(t_{1}\right) \mathrm{d}_{q} s \\
& =k\left(t_{1}, t_{1}\right)(\mathcal{B} y)\left(t_{1}\right)\left[\int_{0}^{t_{2}}\left(t_{2}-q s\right)^{(\beta-1)} \mathrm{d}_{q} s-\int_{0}^{t_{1}}\left(t_{1}-q s\right)^{(\beta-1)} \mathrm{d}_{q} s\right] \\
& =k\left(t_{1}, t_{1}\right) \frac{t_{2}^{(\beta)}-t_{1}^{(\beta)}}{[\beta]_{q}}(\mathcal{B} y)\left(t_{1}\right) \geq 0 .
\end{aligned}
$$

Inequalities (50) and (51) imply that

$$
\int_{0}^{t_{2}} k\left(t_{2}, s\right)\left(t_{2}-q s\right)^{(\beta-1)}(\mathcal{B} y)(s) \mathrm{d}_{q} s-\int_{0}^{t_{1}} k\left(t_{1}, s\right)\left(t_{1}-q s\right)^{(\beta-1)}(\mathcal{B} y)(s) \mathrm{d}_{q} s \geq 0 .
$$

This inequality and (47) gives us

$$
\begin{aligned}
\mid & (\mathcal{T} y)\left(t_{2}\right)-(\mathcal{T} y)\left(t_{1}\right) \mid-\left[(\mathcal{T} y)\left(t_{2}\right)-(\mathcal{T} y)\left(t_{1}\right)\right] \\
\leq & \left\{\left|f\left(t_{2}, y\left(t_{2}\right)\right)-f\left(t_{1}, y\left(t_{1}\right)\right)\right|-\left[f\left(t_{2}, y\left(t_{2}\right)\right)-f\left(t_{1}, y\left(t_{1}\right)\right)\right]\right\} \\
& +\left\{\left|(\mathcal{A} y)\left(t_{2}\right)-(\mathcal{A} y)\left(t_{1}\right)\right|-\left[(\mathcal{A} y)\left(t_{2}\right)-(\mathcal{A} y)\left(t_{1}\right)\right]\right\} \\
& \times \frac{1}{\Gamma_{q}(\beta)} \int_{0}^{t_{2}} k\left(t_{2}, s\right)\left(t_{2}-q s\right)^{(\beta-1)}(\mathcal{B} y)(s) \mathrm{d}_{q} s \\
\leq & d(F y)+\frac{k^{*} \phi\left(r_{0}\right)}{\Gamma_{q}(\beta+1)} d(\mathcal{A} y) .
\end{aligned}
$$

The above estimate implies that

$$
d(\mathcal{T} y) \leq c d(y)+\frac{k^{*} \phi\left(r_{0}\right)}{\Gamma_{q}(\beta+1)} d(\mathcal{A} y) .
$$

Therefore,

$$
d(\mathcal{T Y}) \leq c d(Y)+\frac{k^{*} \phi\left(r_{0}\right)}{\Gamma_{q}(\beta+1)} d(\mathcal{A} Y)
$$

Step 7: $\mathcal{T}$ is contraction with respect to the measure of noncompactness $\mu$. Inequalities (46) and (54) give us that

$$
\omega_{0}(\mathcal{T Y})+d(\mathcal{T Y}) \leq c\left(\omega_{0}(Y)+d(Y)\right)+\frac{k^{*} \phi\left(r_{0}\right)}{\Gamma_{q}(\beta+1)}\left(\omega_{0}(\mathcal{A} Y)+d(\mathcal{A} Y)\right)
$$

or

$$
\mu(\mathcal{T Y}) \leq c \mu(Y)+\frac{k^{*} \phi\left(r_{0}\right)}{\Gamma_{q}(\beta+1)} \mu(\mathcal{A} Y) \leq\left(c+\frac{\eta k^{*} \phi\left(r_{0}\right)}{\Gamma_{q}(\beta+1)}\right) \mu(Y) .
$$

But $c+\frac{\eta k^{*} \phi\left(r_{0}\right)}{\Gamma_{q}(\beta+1)}<1$, then

$$
\mu(\mathcal{T Y}) \leq \mu(Y) .
$$


Inequality (57) enables us to use Theorem 1, then there are solutions to Equation (1) in $C(I)$.

This finishes our proof.

\section{References}

[1] Jackson, F.H. (1910) On q-Definite Integrals. The Quarterly Journal of Pure and Applied Mathematics, 41, $193-203$.

[2] Agarwal, R.P. (1969) Certain Fractional $q$-Integrals and $q$-Derivatives. Proceedings of the Cambridge Philosophical Society, 66, 365-370. http://dx.doi.org/10.1017/S0305004100045060

[3] Jleli, M., Mursaleen, M. and Samet, B. (2016) Q-Integral Equations of Fractional Orders. Electronic Journal of Differential Equations, 2016, 1-14. http://dx.doi.org/10.1186/s13662-015-0739-5

[4] Kac, V. and Cheung, P. (2002) Quantum Calculus. Springer-Verlag, New York. http://dx.doi.org/10.1007/978-1-4613-0071-7

[5] Annaby, M.H. and Mansour, Z.S. (2012) $q$-Fractional Calculus and Equations. Lecture Notes in Mathematics, Springer, Heidelberg, 2056.

[6] Abdeljawad, T. and Baleanu, D. (2011) Caputo $q$-Fractional Initial Value Problems and a $q$-Analogue Mittag-Leffler Function. Communications in Nonlinear Science and Numerical Simulation, 16, 4682-4688. http://dx.doi.org/10.1016/j.cnsns.2011.01.026

[7] Salahshour, S., Ahmadian, A. and Chan, C.S. (2015) Successive Approximation Method for Caputo $q$-Fractional IVPs. Communications in Nonlinear Science and Numerical Simulation, 24, 153-158. http://dx.doi.org/10.1016/j.cnsns.2014.12.014

[8] Banaś, J., Lecko, M. and El-Sayed, W.G. (1998) Existence Theorems of Some Quadratic Integralequations. Journal of Mathematical Analysis and Applications, 222, 276-285. http://dx.doi.org/10.1006/jmaa.1998.5941

[9] Banaś, J., Caballero, J., Rocha, J. and Sadarangani, K. (2005) Monotonic Solutions of a Class of Quadratic Integral Equations of Volterra Type. Computers \& Mathematics with Applications, 49, 943-952. http://dx.doi.org/10.1016/j.camwa.2003.11.001

[10] Caballero, J., Lopez, B. and Sadarangani, K. (2005) On Monotonic Solutions of an Integral Equation of Volterra Type with Supremum. ournal of Mathematical Analysis and Applications, 305, 304-315. http://dx.doi.org/10.1016/j.jmaa.2004.11.054

[11] Darwish, M.A. (2007) On Solvability of Some Quadratic Functional-Integral Equation in Banach Algebra. Communications in Applied Analysis, 11, 441-450.

[12] Darwish, M.A. (2007) On a Singular Quadratic Integral Equation of Volterra Type with Supremum. IC/2007/071, Trieste, Italy, 1-13.

[13] Darwish, M.A. (2008) On Existence and Asympototic Behaviour of Solutions of a Fractional Integral Equation with Linear Modification of the Argument. arXiv: 0805.1422v1.

[14] Darwish, M.A. (2008) On Monotonic Solutions of a Singular Quadratic Integral Equation with Supremum. Dynamic Systems and Applications, 17, 539-550.

[15] Banaś, J. and Rzepka, B. (2007) Monotonic Solutions of a Quadratic Integral Equation of Fractional Order. Journal of Mathematical Analysis and Applications, 332, 1370-1378.

[16] Banaś, J. and O'Regan, D. (2008) On Existence and Local Attractivity of Solutions of a Quadratic Integral Equation of Fractional Order. Journal of Mathematical Analysis and Applications, 345, 573-582. http://dx.doi.org/10.1016/j.jmaa.2008.04.050

[17] Darwish, M.A. (2005) On Quadratic Integral Equation of Fractional Orders. ournal of Mathematical Analysis and Applications, 311, 112-119. http://dx.doi.org/10.1016/i.jmaa.2005.02.012

[18] Darwish, M.A. and Ntouyas, S.K. (2009) Monotonic Solutions of a Perturbed Quadratic Fractional Integral Equation, Nonlinear Analysis: Theory. Methods and Applications, 71, 5513-5521.

[19] Banaś, J. and Olszowy, L. (2001) Measures of Noncompactness Related to Monotonicity. Commentationes Mathematicae, 41, 13-23.

[20] Bhaskar, T.G., Lakshmikantham, V. and Leela, S. (2009) Fractional Differential Equations with a Krasnoselskii-Krein Type Condition. Nonlinear Analysis: Hybrid Systems, 3, 734-737. http://dx.doi.org/10.1016/j.nahs.2009.06.010

[21] Rajković, P.M., Stanković, S.D. and Miomir, S. (2007) Fractional Integrals and Derivatives in q-Calculus. Applicable Analysis and Discrete Mathematics, 1, 311-323. http://dx.doi.org/10.2298/AADM0701311R

[22] Banaś, J. and Goebel, K. (1980) Measures of Noncompactness in Banach Spaces. Lecture Notes in Pure and Applied Mathematics. Marcel Dekker, New York, 60.

[23] Dugundji, J. and Granas, A. (1982) Fixed Point Theory. Monografie Mathematyczne, PWN, Warsaw. 


\section{Submit or recommend next manuscript to SCIRP and we will provide best service for you:}

Accepting pre-submission inquiries through Email, Facebook, Linkedin, Twitter, etc A wide selection of journals (inclusive of 9 subjects, more than 200 journals)

Providing a 24-hour high-quality service

User-friendly online submission system

Fair and swift peer-review system

Efficient typesetting and proofreading procedure

Display of the result of downloads and visits, as well as the number of cited articles

Maximum dissemination of your research work

Submit your manuscript at: http://papersubmission.scirp.org/ 